\title{
Fair Bandwidth Sharing in Distributed Systems: A Game-Theoretic Approach
}

\author{
Sudipta Rakshit and Ratan K. Guha, Member, IEEE
}

\begin{abstract}
Fair sharing of bandwidth remains an unresolved issue for distributed systems. In this paper, the users of a distributed LAN are modeled as selfish users with independence to choose their individual strategies. With these selfish users, the contention-based distributed medium access scenario is modeled as a complete-information, noncooperative game, designated the "Access Game." A novel MAC strategy based on p-persistent CSMA is presented to achieve fairness in the "Access Game." It is proven that there are an infinite number of Nash Equilibria for the "Access Game," but they do not result in fairness. Therefore, it may be beneficial for the selfish users to adhere to a set of constraints that result in fairness in a noncooperative fashion. This leads to the formulation of a constrained "Access Game" with fairness represented as a set of algebraic constraints. It is proven that the solution of the constrained game, the Constrained Nash Equilibrium, is unique. Further, it is shown that, in addition to achieving fairness, this solution also optimizes the throughput. Finally, these results are extended to a more realistic incomplete-information scenario by approximating the incomplete-information scenario as a complete-information scenario through information gathering and dissemination.
\end{abstract}

Index Terms-Distributed systems, local area networks, fair bandwidth share, selfish users, game theory.

\section{INTRODUCTION}

$\mathrm{B}$ ANDWIDTH is one of the primary resources in computer communication networks and Quality of Service (QoS) is influenced by how bandwidth is allocated (in centralized systems) or shared (in distributed systems). As access to bandwidth is dependent on the Medium Access Control (MAC) protocol being used, the research on bandwidth fairness has focused on devising efficient MAC protocols to achieve fairness. A considerable amount of work has been done in this regard. Here, we briefly summarize it.

\subsection{Previous Work}

There are two types of MAC protocols: centralized and distributed. In centralized networks, fairness is based on the concept of Generalized Processor Sharing (GPS). Informally, GPS guarantees a user resource allocation proportional to that user's relative weightage [38]. We will follow this simple, yet powerful definition of fairness for the present work also. GPS cannot be implemented in practice because it relies on bit-by-bit switching, whereas the communication entity of interest is a packet. In [29], a practical packet-based implementation of GPS is presented. This algorithm is usually known as the Weighted Fair Queuing (WFQ) algorithm. In WFQ, each arriving packet is given virtual "start" and "finish" times based on the actual arrival time of the packet and the length of the packet. The packet with the smallest "finish" time is selected for transmission. A similar technique is presented in [39]. There, the WFQ algorithm designated Packet GPS (PGPS) is combined with a Leaky Bucket Admission Control algorithm for a single server GPS and it is shown that it is possible for the network to fulfill a

- The authors are with the School of Computer Science, University of Central Florida, Orlando, FL 32816. E-mail: \{sraks, guha\}@cs.ucf.edu.

Manuscript received 11 June 2004; revised 10 Feb. 2005; accepted 31 May 2005; published online 16 Sept. 2005.

For information on obtaining reprints of this article, please send e-mail to: tc@computer.org, and reference IEEECS Log Number TC-0197-0604. wide range of performance guarantees using these algorithms. In [40], an improved GPS approximation algorithm, called Worst-case Fair Weighted Fair Queuing (WF2Q), is proposed. Using WF2Q, only packets with a virtual "start" time that has been passed are considered for transmission. This is a more accurate approximation of GPS, but increases implementation complexity. In distributed systems, there are two main problems in achieving fairness: lack of information, i.e., users usually do not know about the number of other users, and lack of coordination, i.e., one user cannot determine if any other is also transmitting simultaneously. Of these two problems, lack of coordination is more fundamental in nature because, even if the users know about the number of other users present in the system, medium access and packet transmission cannot be coordinated. Therefore, the objective of bandwidth fair sharing in a distributed system is to resolve this contention in such a way that users get bandwidth proportional to their weightages. For distributed systems, most of the work concentrates on Carrier Sense Multiple Access (CSMA) MAC protocols. In [35], a Distributed Fair Scheduling (DFS) scheme based on a virtual clock mechanism has been proposed for a Wireless Local Area Network (WLAN). As in WFQ, the "start" and "finish" times of an arriving packet are computed and the packet with the smallest "finish" tag is transmitted. A distributed algorithm using the back-off interval mechanism of IEEE802.11 MAC [11], [12] is used to determine the packet with the smallest "finish" tag. However, in general, it is difficult to implement a "virtual clock" mechanism in distributed systems. Another approach in differentiated bandwidth sharing in distributed systems is the "priority-based" access schemes. One of the earliest works incorporating priority in CSMA can be found in [41]. There, Tobagi presented a prioritized CSMA or P-CSMA. ${ }^{1}$ The idea behind this scheme is as follows: grant access right exclusively to the messages of the current

1. Different from $p$-CSMA. 
highest priority class. In [33], priority-based access schemes using the Carrier Sense Multiple Access (CSMA) protocol are analyzed for 1-persistent and nonpersistent modes. p-persistence CSMA is not considered due to the difficulty in analysis. Specifically, three schemes are considered: 1) all packets transmitted in 1-persistent mode, 2) higher priority packets transmitted in 1-persistent mode and lower priority packets transmitted in nonpersistent mode, and 3) all packets transmitted in nonpersistent mode. Assuming Poisson packet arrival and general packet length distributions mean packet delays are computed using approximate techniques. Another priority-based scheme for CSMA is presented in [34]. Some other related work for distributed systems can be found in [31] and [32]. The problem with the "priority-based" access schemes is the lack of an explicit relation between and priority and fairness. The medium access strategy we proposed is philosophically similar to the priority-based approach, but completely different in modeling and analysis.

\subsection{Motivation and Contribution}

The drawbacks of both the "virtual clock" and "prioritybased" schemes have been indicated. Here, we present a novel approach for contention resolution by modeling the contention for medium access as a Non-Cooperative Game, the "Access Game." The "Access Game" model is predicated on an explicit relation between priority and fairness. Therefore, the solution of the "Access Game" satisfies the fairness definition as enunciated by GPS. Game Theory has been extensively used in other areas of computer communication [15], [16], [17], [18], [19], [20], [21]. However, there has been only limited application of Game Theory for designing distributed MAC protocols. It was recently introduced in [13]. To the best of the authors' knowledge, the present work is the first attempt to formulate the fairness problem in a Game-Theoretic framework. There are two reasons why Game Theory is a suitable tool for analyzing distributed medium access. First, the contention-based nature of medium access presents a natural application domain for Non-Cooperative Game Theory. Second, it is possible to conceive of "selfish" users in the future choosing their individual access strategies to optimize their own selfish interests [13], [37], [30]. The "virtual time"-based or "static priority"-based approaches described above are not suited for such situations. The "Access Game" model provides a theoretical formulation for achieving fair bandwidth sharing in the presence of "selfish" users. In addition to resolving the fairness problem, we also investigate in detail the interaction between the optimal "selfish" user strategies and the overall system performance.

\subsection{Organization}

The rest of the paper is organized as follows: Section 2 briefly describes some Game-Theoretic concepts and provides the "Access Game" model for the contention-based medium access, Section 3 analyzes a hypothetical completeinformation scenario and proves the uniqueness of transmission strategies achieving fairness, Section 4 provides techniques to approximate an incomplete information scenario to a complete information scenario and shows that fair bandwidth share is achieved in this approximate case also, and Section 5 concludes this paper.

\section{Modeling the Access Game}

Formally, a finite game $G$ consists of a nonempty finite set $I$ of players. A player, say $i$, has a set of possible strategies/ actions $A_{i}$. In order to play the game, all the players choose an action from the respective strategy sets simultaneously. At the end of the game, there is an outcome. Clearly, the outcome space is given by $S=\times_{i} A_{i}$. Let $s \in S$ be a generic outcome of the game $G$. Associated with the outcome, $s$, is a payoff to each of the players. Let us designate by $u_{i}=u_{i}(s)$ the payoff function for the $i$ th user. The payoff function of the game is an ordered tuple of payoffs to individual users and is given by $u(s)=\left(u_{1}(s) \ldots u_{n}(s)\right)$. Initially, Game Theory concentrated on games of pure strategy, i.e., user $i$ taking only one action with probability " 1 " to play the game. Nash introduced the concept of mixed strategy. The concept of "mixed strategy" is that, instead of deciding for a particular action with certainty, a user $i$ randomizes its decision and chooses a particular action from $A_{i}$ with a probability (may be zero). Consequently, the elements of the outcome set $S$ also become probabilistic in nature. As the payoffs are associated with the outcome of the game, it follows that, in a mixed strategy game, there is a nonnegative probability attached to the value of the payoff a user receives by playing the game. This entails the formulation of utility function $\bar{u}_{i}$ for each player $i$. In a mixed strategy game $G$, utility function $\bar{u}_{i}$ is the expected payoff for player $i$. We consider two solution concepts of a Non-Cooperative Game: Nash Equilibrium [26] and Constrained Nash Equilibrium [28]. For detailed discussion on these topics, see [14].

\subsection{Access Game}

Examples of distributed MAC protocols are ALOHA [3], Carrier Sense Multiple Access/Collision Detection (CSMA/ CD) [5], [6], [7], [8], [9], and Carrier Sense Multiple Access / Collision Avoidance (CSMA/CA) [10], [11], [12], whereas an example of centralized MAC protocol is HIPERLAN [1], [2]. For the present work, a $p$-CSMA type access strategy for medium access is considered. Before proceeding further, a brief description on the $p$-CSMA protocol is in order. Consider a MAC protocol using CSMA. When a user has a packet to send, it senses the medium and, if the medium is sensed busy, it can take several actions and, depending on these actions, CSMA protocols can be classified as follows [4]:

1. 1-persistent: Keep on sensing the medium and transmit the packet when the channel becomes idle.

2. Non-persistent: Do not sense the medium for some time (i.e., back-off).

3. $p$-persistent: Keep on sensing the medium as long as the medium is busy and when the medium becomes idle, transmit with probability $p$ and wait with probability (1-p).

We provide the following diagram (Fig. 1) for a schematic representation of $p$-CSMA.

It can be observed from Fig. 1 that, at the end of a transmission period, there is a brief idle period. The idle period essentially signals the end of the previous 


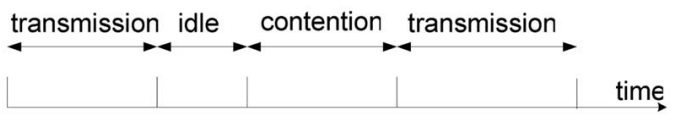

Fig. 1. Successive states of the system in p-CSMA.

transmission period. After this brief idle period, users contend with each other to access the medium. Contention is eventually resolved in one user's favor and the successful user transmits next.

The MAC strategy presented for the "Access Game" is similar to the $p$-CSMA. The significant difference is that the value of " $p$ " is not constant in the proposed protocol. Users compute appropriate values of $p$, based on the state of the system. More specifically, users transmit with different probabilities depending on the number of users present in the system. These are the players of the "Access Game." At the beginning of each contention period, each player has two actions to choose from: "transmit" and "wait." User $i$ transmits with probability $p_{i}$. The game has three outcomes: "success," "failure," and "waste." The outcomes and payoffs of the game are as follows:

1. If no user transmits, the game's outcome is "waste" and all the users receive a payoff of " 0. "

2. If exactly one user transmits (say user $i$ ), then the outcome of the game is "success." User $i$ receives a payoff of " 1 " and all the other users receive a payoff of " 0. "

3. If more than one user transmits, collision occurs and the outcome of the game is "failure." Every user receives a payoff of " $0 . "$

Note that if a user decides to "wait," irrespective of the game's outcome, it receives a payoff of "0." This choice of payoff is justified by the selfish nature of the users. Let us give a simple example regarding the payoff: Consider two users $\mathrm{A}$ and $\mathrm{B}$ contending for transmission. If both of them transmit, there is a collision and nobody benefits; hence, a payoff of " 0 " is assigned to both the users. If A (or B) decides to wait, it may so happen that $\mathrm{B}$ (or A) transmits and benefits. However, no benefit is accrued to A (or B). Therefore, A (or B) receives a payoff of "0." The payoff of " 1 " for success is self-explanatory.

Following the above discussion, the utility function or the expected payoff function can be expressed as:

$$
\bar{u}_{i}=\operatorname{Pr}(\text { success }) \times 1 .
$$

From here on, we drop the "bar" in $\bar{u}_{i}$ and denote the payoff function of user $i$ as simply $u_{i}$. The main results are now presented in the following section.

\subsection{Social Welfare}

We have modeled the medium access as a Non-Cooperative Game. In the next section, solutions to the "Access Game" are provided. Before presenting these solutions, we briefly discuss some desirable qualities of these solutions in terms of Social Welfare and Pareto Optimality. Social Welfare (SW) is a concept from economics dealing with the distribution of resources in the society. Interestingly, this concept can be applied in the present context of medium access also, with the resource in question being the bandwidth. We have previously argued that each user should receive their fair share of bandwidth. Therefore, SW for the "Access Game" is achieved if these fairness criteria are satisfied. Associated with the concept of social welfare is the concept of Pareto-Optimality (PO) of a resource allocation. Informally, PO is achieved if nobody can be made better off without making somebody else worse off. All PO solutions do not result in SW, but all the allocations resulting in SW are PO. Whether the PO solutions satisfy the condition of SW depends on how SW is defined in a particular context. As the solutions in the next section satisfy SW within the present context, the solutions are PO in nature as well.

\section{Analysis of the Access Game}

From the discussion in the previous section, it is clear that each user has only one decision variable: the probability to transmit. In this section, we present the main results regarding these decision variables. First, the assumptions for the analysis conducted in this section are specified.

\subsection{Assumptions}

A1. Each user has complete information about all the other users. Although this assumption is quite restrictive for a distributed system, it is made to provide a sharp analysis. This assumption is later relaxed.

A2. All the users have packets to transmit. This assumption is made for the sake of simplicity. We also relax this assumption in the next section.

A3. Packets are of equal length. This assumption is again made for simplicity.

A4. The number of users playing the game is $n$ and this number does not change. As $n=1$ presents a trivial case, $n>1$ is assumed.

A5. The system is stable.

Assumptions 4 and 5 are related. In this section, we present the results for a fixed number of users. In the next section, we relax this assumption and allow for varying numbers of users. For our analysis to hold for the general case in the next section, the system needs to be stable. The stability assumption has been maintained throughout this paper.

\subsection{Nash Equilibrium}

We first prove that the Nash Equilibria for the "Access Game" is inefficient.

Theorem 1. In the Nash Equilibrium for the Access Game, there is at least one user $i$ such that $p_{i}^{N E}=1$.

Proof. Reproducing the utility function of user $i$ from (1), we have

$$
u_{i}=p_{i} \prod_{j \neq i}^{n}\left(1-p_{j}\right) .
$$

Of interest is the expression $\prod_{j \neq i}^{n}\left(1-p_{j}\right)$. Let us call it $\delta_{i}$ :

$$
\delta_{i}=\prod_{j \neq i}^{n}\left(1-p_{j}\right) .
$$




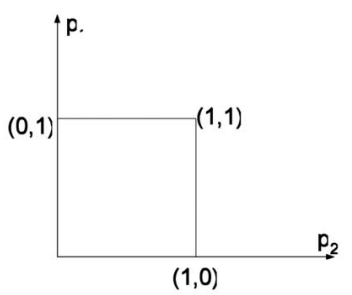

(a)

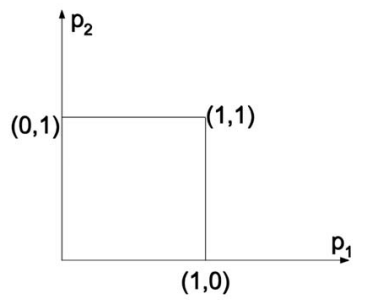

(b)

Fig. 2. (a) Best response in $p_{1}$ as a function of $p_{2}$. (b) Best response in $p_{2}$ as a function of $p_{1}$.

Clearly,

$$
\delta_{i} \geq 0 \quad \forall i
$$

Observing (2) and following the example in the Appendix, which can be found on the Computer Society Digital Library at http:/ / computer.org/tc/archives.htm, it can be said that

when $\delta_{i}>0$, i.e., $p_{j} \neq 1 \forall j \neq i, u_{i}$ is maximized by $p_{i}=1$

when $\delta_{i}=0$, i.e., $\exists j \neq i: p_{j}=1$,

$u_{i}$ is maximized by $p_{i}=\{x: x \in(0,1)\}$

(i.e., any value in the range between 0 and 1 ).

Note that the noncooperative users do not communicate with each other about their strategy. An NE is achieved when users simultaneously maximize their utility functions in such a noncooperative fashion.

We now prove the theorem by contradiction. Let us assume that, at the NE,

$$
p_{i}^{N E} \neq 1 \quad \forall i
$$

It immediately follows that, at the NE, $\delta_{i}>0 \forall i$ and, by (4.a),

$$
p_{i}^{N E}=1 \quad \forall i .
$$

It is easy to see that (5.a) and (5.b) are clear contradictions. Therefore, our assumption that $p_{i}^{N E} \neq 1 \forall i$ is not correct.

We now explain Theorem 1 with the help of the $n=2$ case. Following (4.a) and (4.b), we have plotted the best response in $p_{1}$ as a function of $p_{2}$ in Fig. 2a. As a way of explanation, let us start at $p_{2}=0$. The value of $p_{1}$ is " 1 " at $p_{2}=0$. The value of $p_{1}$ remains " 1 " as long as $p_{2}<1$. At $p_{2}=1$, the best response in $p_{1}$ is any value in the interval
$(0,1)$, i.e., there are an infinite number of best responses in $p_{1}$ for $p_{2}=0$. This is represented by the straight line joining $(1,1)$ and $(0,1)$.

In Fig. $2 b$, we have plotted the best response in $p_{2}$ as a function of $p_{1}$. It can clearly be seen that the best response plots of the two players completely overlap. As the NE are given by the intersections of the best response correspondences, there is an infinite number of NE for the "Access Game." It is not difficult to compute the various Nash Equilibriums of the "Access Game." For the example of $n=$ 2 users, if user 1 is transmitting at $p_{1}=1$, user 2 can transmit with any probability from 0 to 1 in order to maximize its own utility function. On the other hand, if user 2 is transmitting with any probability from $0 \leq p_{2}<1$, the utility function of user 1 is maximized by $p_{1}=1$. Therefore, one set of NE is given by $\left\{\left(1, p_{2}\right): 0 \leq p_{2}<1\right\}$. Similarly, if $p_{2}=1$, the utility of the user is maximized by $0 \leq p_{1}<1$. Therefore, the other set of NE is given by $\left\{\left(p_{1}, 1\right): 0 \leq p_{1}<1\right\}$. Finally, we have $(1,1)$ as an NE also.

From this discussion, it can be noted that there is an infinite number of NE and, for each of the NE, at least one of the users has a transmission probability of 1 . It follows that the success probability of at least one of the users would be " 0 ."

Corollary 2. There are infinite numbers of NE for the "Access Game."

Proof. Follows from Theorem 1 and the discussion presented for $n=2$.

Definition 1. A solution of the Access Game is acceptable if and only if the probability of success is nonzero for all the users.

Using Definition 1, we have the following result:

Corollary 3. Nash Equilibria for the Access Game are not acceptable.

Proof. There are two possible cases.

Case 1: Only one user $i$ is transmitting with $p_{i}=1$. $\operatorname{Pr}\{$ success $\}=0$ for all the other users.

Case 2: More than one user is transmitting with probability "1." Hence, $\operatorname{Pr}\{$ success $\}=0$ for all the users.

From the above results, we see that, although an infinite number of Nash Equilibria exist, they are inefficient. Therefore, the rational users would be willing to adhere to a set of constraints if these constraints benefit them.

\subsection{Constrained Nash Equilibrium}

Our objective here is to achieve fairness in bandwidth sharing and, from a selfish user's point of view, it will be an attractive proposition if some set of constraints leads to fairness in accessing the medium; more so because completely independent actions produce inefficient equilibria (as proven above). We now investigate the feasibility of solution with a set of constraints [42].

Before proceeding further, we reproduce Theorem 1 of [28] for completeness and ready reference.

Theorem 4. An equilibrium exists for every concave n-person game. 
For Theorem 4 to hold, the utility functions of all the users should be concave in nature with respect to their respective strategies. The striking characteristic of this theorem is that the strategy spaces of different users need not be orthogonal (independent). For such strategies, Theorem 4 ensures an equilibrium if the utility functions are concave. From (1), we have $\frac{\partial^{2} u_{i}}{\partial p^{2}}=0$. Therefore, $u_{i}$ is concave in $p_{i}$ and we can apply Theorem 4 to our problem.

In order to derive our following results, we now present the concept of fairness as a precise set of constraints. In the present context of distributed medium access, the fairness is defined as follows [24]:

Definition 2. Fairness is achieved if the probability that user $i$ accesses the medium is proportional to its weightage $w_{i}(>0)$.

Hence, fairness can be quantitatively expressed as

$$
\frac{p_{1} \prod_{j \neq 1}^{n}\left(1-p_{j}\right)}{w_{1}}=\ldots \frac{p_{i} \prod_{j \neq i}^{n}\left(1-p_{j}\right)}{w_{i}}=\ldots \frac{p_{n} \prod_{j=1}^{n-1}\left(1-p_{j}\right)}{w_{n}} .
$$

As we are interested in only nontrivial and acceptable solutions,

$$
p_{i} \neq 0,1 \quad \forall i .
$$

It should be noted that the CNEs can be computed without the condition in (7), but we are interested only in the solutions satisfying (7).

Combining (6) and (7), we have

$$
\frac{p_{1} /\left(1-p_{1}\right)}{w_{1}}=\ldots \frac{p_{i} /\left(1-p_{i}\right)}{w_{i}}=\ldots \frac{p_{n} /\left(1-p_{n}\right)}{w_{n}}=\frac{1}{K},
$$

where $K$ is a positive constant, i.e.,

$$
\begin{gathered}
K>0, \\
\text { therefore }\left[\frac{1}{p_{i}}-1\right]=\frac{w_{j}}{w_{i}}\left[\frac{1}{p_{j}}-1\right] \quad \forall i, j .
\end{gathered}
$$

From the above discussions and Theorem 4 , we have the following lemma:

Lemma 5. A CNE exists for the Access Game satisfying the fairness condition in (8).

With the previous formulation, the following theorem is proven.

Theorem 6. At the CNE satisfying the fairness conditions in (8), the transmission probabilities satisfy the following:

$$
\sum_{i=1}^{n} p_{i}=1
$$

Proof. Using (2) and (7), we have

$$
u_{i}=p_{i} \prod_{j \neq i}^{n}\left(1-p_{j}\right)=\frac{p_{i}}{1-p_{i}} g
$$

where $g=\prod_{j=1}^{n}\left(1-p_{j}\right)$ and, from (7),

$$
g=\prod_{j=1}^{n}\left(1-p_{j}\right) \neq 0 .
$$

For the "Access Game" CNE satisfying the fairness conditions, we have $\frac{\partial u_{i}}{\partial p_{i}}=0$ [28] and the fairness conditions:

$$
\frac{p_{1} /\left(1-p_{1}\right)}{w_{1}}=\ldots \frac{p_{i} /\left(1-p_{i}\right)}{w_{i}}=\ldots \frac{p_{n} /\left(1-p_{n}\right)}{w_{n}}=\frac{1}{K} .
$$

From $\frac{\partial u_{i}}{\partial p_{i}}=0$, we have, using (11),

$$
g \frac{\partial\left(\frac{p_{i}}{1-p_{i}}\right)}{\partial p_{i}}+\frac{p_{i}}{1-p_{i}} \frac{\partial g}{\partial p_{i}}=0 .
$$

From (10), we have $\frac{p_{i} / 1-p_{i}}{w_{i}}=\frac{p_{j} / 1-p_{j}}{w_{j}} \forall i, j$.

It follows that $\frac{\partial p_{j}}{\partial p_{i}}=\frac{p_{j}^{2}}{p_{i}^{2}} \times \frac{w_{i}}{w_{j}}=\frac{p_{j}^{2}}{p_{i}^{2}} \times \frac{p_{i}}{1-p_{i}} \times \frac{1-p_{j}}{p_{j}}$ :

$$
\text { therefore } \frac{\partial p_{j}}{\partial p_{i}}=\frac{p_{j}}{1-p_{i}} \times \frac{1-p_{j}}{p_{i}} \text {. }
$$

We compute $\frac{\partial g}{\partial p_{i}}$ by using (14).

In order to demonstrate how the proof works, we first prove the case of two users. We have

$$
g \frac{\partial\left[\frac{p_{1}}{1-p_{1}}\right]}{\partial p_{1}}+\frac{p_{1}}{1-p_{1}} \frac{\partial g}{\partial p_{1}}=0,
$$

$$
\begin{aligned}
& g=\left(1-p_{1}\right)\left(1-p_{2}\right) \\
& \frac{\partial g}{\partial p_{1}}=-\left[\left(1-p_{2}\right)\right]+\left(1-p_{1}\right)\left[\frac{\partial}{\partial p_{1}}\left(1-p_{2}\right)\right] .
\end{aligned}
$$

The chain partial differentiation in the next step is the "trick" in this proof:

$$
\frac{\partial g}{\partial p_{1}}=-\left[\left(1-p_{2}\right)+\left(1-p_{1}\right)\left[\left\{\frac{\partial}{\partial p_{2}}\left(1-p_{2}\right)\right\} \frac{\partial p_{2}}{\partial p_{1}}\right] .\right.
$$

From (14), $\frac{\partial p_{2}}{\partial p_{1}}=\frac{p_{2}}{1-p_{1}} \times \frac{1-p_{2}}{p_{1}}$,

$$
\text { therefore } \frac{\partial g}{\partial p_{1}}=-\left[\left(1-p_{2}\right)+\left(1-p_{1}\right) \frac{p_{2}\left(1-p_{2}\right)}{p_{1}\left(1-p_{1}\right)}\right]
$$

or

$\frac{\partial g}{\partial p_{1}}=-\left[\left(1-p_{2}\right) \frac{p_{1}}{p_{1}}+\frac{p_{2}\left(1-p_{2}\right)}{p_{1}}\right]=-\frac{\left(1-p_{2}\right)}{p_{1}}\left[p_{2}+p_{1}\right]$,

therefore $\frac{\partial g}{\partial p_{1}}=-\frac{g}{p_{1}\left(1-p_{1}\right)}\left[p_{2}+p_{1}\right]=-\frac{g}{p_{1}\left(1-p_{1}\right)} \sum_{i=1}^{2} p_{i}$.

Using (16) in (15) and the fact that $\frac{\partial\left(p_{i} / 1-p_{i}\right)}{\partial p_{i}}=\frac{1}{\left(1-p_{i}\right)^{2}}$, we have

$$
\frac{g}{\left(1-p_{1}\right)^{2}}-\frac{p_{1}}{1-p_{1}} \times \frac{g}{p_{1}\left(1-p_{1}\right)} \times\left(\sum_{i=1}^{2} p_{i}\right)=0 .
$$


From (12), $g \neq 0$,

$$
\text { therefore } \sum_{i=1}^{2} p_{i}=1 \text {. }
$$

For a general case involving $n$ users, following the exact same steps and the chain differentiation trick, we have

$$
g \frac{\partial\left(\frac{p_{i}}{1-p_{i}}\right)}{\partial p_{i}}+\frac{p_{i}}{1-p_{i}} \frac{\partial g}{\partial p_{i}}=0
$$

and

$$
\frac{\partial g}{\partial p_{i}}=-\frac{g}{p_{i}\left(1-p_{i}\right)} \sum_{i=1}^{n} p_{i}
$$

Hence, we have

$$
\sum_{i=1}^{n} p_{i}=1
$$

We now prove that there is a unique solution for probabilities satisfying (8) and (17).

Theorem 7. The CNE for the Access Game satisfying the fairness conditions in (8) is unique.

Proof. From (8), we have

$$
p_{i}=\frac{w_{i}}{K+w_{i}} \quad \forall i .
$$

Using (17) and (18), we have

$$
\sum_{i=1}^{n} \frac{w_{i}}{K+w_{i}}=1
$$

We show that the solution for $K$ in (19) is unique. We prove the uniqueness of the solution in three parts.

1. All the solutions of $K$ in (19) are real.

Proof. Let there be complex solution for $K$; $\alpha \pm \sqrt{-1} \beta$. We then have

$$
\begin{gathered}
\begin{array}{c}
\sum_{i=1}^{n} \frac{w_{i}}{\alpha+\sqrt{-1} \beta+w_{i}}=1 \text { and } \sum_{i=1}^{n} \frac{w_{i}}{\alpha-\sqrt{-1} \beta+w_{i}}=1 \\
\text { therefore } \sum_{i=1}^{n}\left(\frac{w_{i}}{\alpha+\sqrt{-1} \beta+w_{i}}-\frac{w_{i}}{\alpha-\sqrt{-1} \beta+w_{i}}\right) \\
=0
\end{array} \\
\text { therefore }-2 w_{i} \sqrt{-1} \beta \sum_{i=1}^{n} \frac{1}{\left(\alpha+w_{i}\right)^{2}+\beta^{2}}=0 . \\
\text { As } \sum_{i=1}^{n} \frac{w_{i}}{\left(\alpha+w_{i}\right)^{2}+\beta^{2}} \neq 0, \\
\beta=0 .
\end{gathered}
$$

2. There is at least one positive root for $K$.

Proof. Equation (19) has $n$ solutions in $K$. Let these roots be $r_{1} \ldots r_{n}$.
By expanding (19), it can be clearly seen that some of the coefficients are negative. Hence, there is at least one positive root. Formally,

$$
\exists k: r_{k}>0 .
$$

3. If the number of positive roots in (19) is more than one, they are equal.

Proof. Let there be more than one positive root; $\alpha_{1}, \alpha_{2}$. From (19), we have:

$$
\begin{aligned}
& \sum_{i=1}^{n}\left(\frac{w_{i}}{\alpha_{1}+w_{i}}-\frac{w_{i}}{\alpha_{2}+w_{i}}\right)=0 \\
& \text { therefore }\left(\alpha_{2}-\alpha_{1}\right) \sum_{i=1}^{n} \frac{w_{i}}{\left(\alpha_{1}+w_{1}\right)\left(\alpha_{2}+w_{i}\right)}=0
\end{aligned}
$$

or

$$
\alpha_{2}=\alpha_{1} .
$$

From (20)-(22), we have that the solution of $K$ in (19) is unique. Hence, from (18), the CNE is unique.

A closed form solution for $K$ in $\sum_{i=1}^{n} \frac{K}{K+w_{i}}=1$ cannot be obtained for $n \geq 5$ (Abel's Impossibility Theorem). Values of $K$ are provided for some special cases given below:

1. Two users: $K=\sqrt{w_{1} w_{2}}$.

2. $n$ identical users: $K=(n-1) w$.

3. Two classes $(1,2)$ of users and class $i$ has $n_{i}$ users. Each user of class $i$ has weight $w_{i}: K=\frac{\sqrt{b^{2}+4 a c}-b}{2 a}$, where

$$
\begin{aligned}
& a=\left(n_{1}+n_{2}-1\right), b=w_{2}\left(n_{1}-1\right)+w_{1}\left(n_{2}-1\right), \\
& c=w_{1} w_{2} .
\end{aligned}
$$

\subsection{Throughput}

Next, we prove that the throughput is also optimized at the CNE. The following result is useful in that regard.

Lemma 8. At the CNE satisfying fairness conditions in (8), $\frac{\partial u_{i}}{\partial p_{j}}=$ $0 \forall i, j$.

Proof. For CNE, $\frac{\partial u_{i}}{\partial p_{i}}=0 \forall i$ and $\frac{p_{i} /\left(1-p_{i}\right)}{w_{i}}=\frac{p_{j} /\left(1-p_{j}\right)}{w_{j}} \forall i, j$. We have $u_{i}=\frac{p_{i}}{1-p_{i}} g$.

It follows that, at CNE, $u_{i}=\frac{w_{i}}{w_{j}} \frac{p_{j}}{1-p_{j}} g=\frac{w_{i}}{w_{j}} u_{j}$.

But, at CNE, $\frac{\partial u_{j}}{\partial p_{j}}=0 \forall j$ :

$$
\text { therefore } \frac{\partial u_{i}}{\partial p_{j}}=\frac{w_{i}}{w_{j}} \frac{\partial u_{j}}{\partial p_{j}}=0 \quad \forall i, j .
$$

We now use Lemma 8 to prove that the throughput is optimized at the CNE.

Theorem 9. CNE satisfying fairness maximizes the system throughput. 
Proof. By throughput, we mean the probability that any user succeeds in contention. Then, the system throughput $\theta$ can be given as

$$
\theta=\sum_{i=1}^{n} p_{i} \times \prod_{j \neq i}^{n}\left(1-p_{j}\right) .
$$

From (7), $\theta=\sum_{i=1}^{n} \frac{p_{i}}{1-p_{i}} \times g$.

From (11),

$$
\theta=\sum_{i=1}^{n} u_{i}
$$

For throughput maximization, $\frac{\partial \theta}{\partial p_{j}}=0 \forall j$. From (24),

$$
\frac{\partial \theta}{\partial p_{j}}=\sum_{i=1}^{n} \frac{\partial u_{i}}{\partial p_{j}}
$$

Following Lemma 8 (23), we have

$$
\left.\frac{\partial \theta}{\partial p_{j}}\right|_{C N E}=0 \quad \forall j
$$

It is interesting to note that the transmission probabilities at the CNE have the dual properties of satisfying the fairness conditions and optimizing the throughput. We now discuss this characteristic in some detail. It can be said that the formulation of the "Access Game" in this paper has a fairness-bias, i.e., achieving fairness in a distributed medium access scenario is the primary concern and this concern has been incorporated in the form of the fairness conditions in (8). Therefore, it is no surprise that the proposed solution does indeed result in fairness. What seems a little counterintuitive and surprising is the fact that the throughput is also optimized. It should be noted that the trivially maximal value for the throughput would be " 1 ," but, in such a scenario, fairness would probably be adversely affected. Therefore, in this paper, the throughput is optimized within the constraints of fairness. The qualitative reason behind the optimization is the judicious use of the channel by the users. Analytically, let us look the expression for the throughput: $\theta=\sum_{i=1}^{n} u_{i}$. The solution proposed in this paper (i.e., the CNE) simultaneously optimizes the utility function of all the users within the constraints of the fairness conditions. Therefore, it naturally follows that the throughput would also be similarly optimized. Finally, we state, without proof, that there is a unique set of transmission probabilities that satisfies the fairness conditions and optimize the throughput. It follows that this set of transmission probabilities is identical to the CNE of the "Access Game."

\subsection{Generalized Payoff Scheme}

While formulating the "Access Game" in Section 2, we had assigned a nonzero payoff to "success" only. It was justified because of the selfish nature of the users and no other issue was considered. However, it is plausible that there can be other considerations, e.g., battery power consumption for communication purposes. Consequently, there can be nonzero payoffs associated with other outcomes also. A general framework for such cases is now provided. As before, there are three outcomes for each individual user: "success," "failure," and "waste." Let us designate by $c_{1, i}$, $c_{2, i}$, and $c_{3, i}$ the payoffs to user $i$ for "success," "wait," and "failure," respectively. In order to distinguish the utility function for the general case, we use the subscript "general."

The utility function $u_{i, g e n e r a l}$ for $i$ is given by

$$
\begin{aligned}
u_{i, \text { general }} & =p_{i} \times \prod_{j \neq i}^{n}\left(1-p_{j}\right) \times c_{1, i} \\
& +p_{i}\left[1-p_{i} \times \prod_{j \neq i}^{n}\left(1-p_{j}\right)\right] \times c_{3, i}+\left(1-p_{i}\right) \times c_{2, i} .
\end{aligned}
$$

Equation (26) can be rewritten as follows:

$$
\begin{aligned}
u_{i, \text { general }} & =p_{i} \times\left[\prod_{j \neq i}^{n}\left(1-p_{j}\right) \times c_{1, i}\right. \\
& \left.+\left\{1-p_{i} \times \prod_{j \neq i}^{n}\left(1-p_{j}\right) \times c_{3, i}\right\}-c_{2, i}\right]+c_{2, i} .
\end{aligned}
$$

The quantity within the square brackets is independent of $p_{i}$. Hence, the utility function is concave with respect to $p_{i}$. Using Theorem 4, we have the following lemma.

Lemma 10. A CNE satisfying the fairness criterion of (8) exists for the general payoff scenario described in (27).

A detailed analysis for the general formulation is beyond the scope of this paper. An analysis in the context of wireless networks has been presented in [36].

\section{IMPLEMENTATION}

The results of the previous section were derived under assumptions A1-A5. In this section, most of these assumptions are relaxed.

\subsection{Incomplete Information}

We assumed that the number of users in the system remains fixed. However, in practice, the number of users varies in a LAN. Therefore, we relax this assumption in this section. We also assumed that the users in the system always have packets. However, this is not realistic. We relax this assumption also. As mentioned before, the assumption of stability remains in place.

Finally, we assumed that the "Access Game" is a complete information game. The implication of this assumption was that a user knew about the presence of the other users and their weightages. In a realistic distributed network, this implication will not hold true and, strictly speaking, the "Access Game" should be treated as an incomplete information game [27]. However, the solutions of a complete information game are more efficient in nature. Consequently, we have proposed a simple technique that will make users aware of the number of users present in the system and their weightages. This technique would enable us to approximate an incomplete information scenario as a complete information one. 


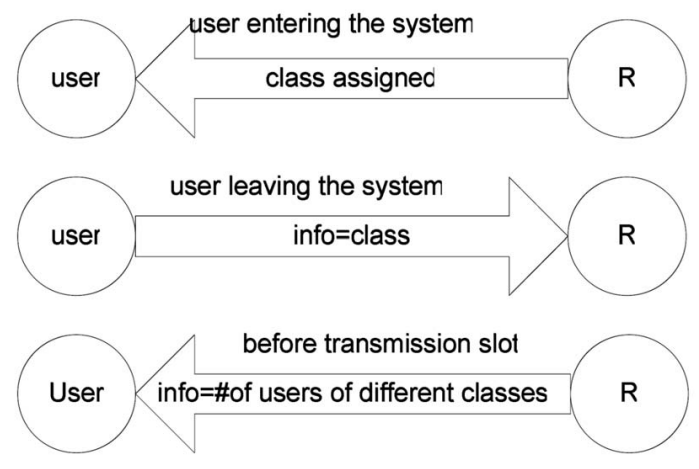

Fig. 3. Approximation scheme appхт.

\subsection{Approximation Scheme}

The key to our approximation process is as follows: In most of the distributed systems, there is usually a registration authority $^{2}$ (henceforth designated by $R$ ) that performs several accounting functions. We use $R$ to gather and disseminate information. It should be noted that using $R$ does not dilute the distributed nature of the medium access in the system. $R$ plays no direct role in medium access by users; it only helps users to make informed decisions.

\subsubsection{Information Gathering and Dissemination}

Let us call the approximation scheme appxm. There are two conceptual steps in appxm. Information is sent to $R$ by users (information gathering) and the information accumulated from all the users is broadcast over the network by $R$ (information dissemination). There is a wide body of work dealing with information gathering and dissemination. Our objective is to provide a simple, case-specific mechanism to achieve approximation. The particulars of the mechanism are as follows:

1. Users are divided into $C$ classes. Associated with a class $c$ are two parameters: weightage, $w_{c}$, and the probability of having a packet at the beginning of a transmission slot, $p_{p, c} \neq 0,1$. Users know these parameters a priori.

2. $R$ maintains a table containing the number of different classes of users in the system.

3. When a user enters the system, it is assigned a class and the number of users in the corresponding class is increased by one. Similarly, when a user leaves the system, it informs $R$ about its decision to leave. The number of users in the corresponding class of users is decreased by one. Separate control channels should be used for the registration and deregistration process.

4. Before the beginning of each transmission slot, $R$ broadcasts the number of users of each class present in the system.

The steps are depicted in Fig. 3.

\subsection{Analysis}

Although we could have introduced the class-specific notations for the following analysis, we have followed the

2. This does not hold for ad hoc networks. notation of Section 3 to show the equivalence of the approximate scenario with a complete information scenario.

When appxm is used, a user knows about the number of other users present in the system. In other words, a user also knows about the values of $w_{i} \mathrm{~s}$ and $p_{p, i} \mathrm{~s}$.

The utility function of user $i$ can be given as (from (1))

$$
u_{i}=p_{i} \times \prod_{j \neq i} p_{W, j},
$$

where $p_{W, j}$ is the probability that user $j$ does not transmit a packet. Note that a user $j$ does not transmit if

1. it does not have a packet to transmit OR

2. it has a packet to transmit, but decides not to transmit.

Therefore, $p_{W, j}$ can be computed as follows:

$$
\begin{aligned}
& p_{W, j}=p_{p, j} \times\left(1-p_{j}\right)+\left(1-p_{p, j}\right)=1-p_{p, j} \times p_{j} \\
& \text { therefore } u_{i}=p_{i} \times \prod_{j \neq i}^{n} p_{w, j}=\frac{p_{i}}{1-P_{p, i} \times p_{i}} \times \prod_{j=i}^{n} p_{w, j} .
\end{aligned}
$$

It is clear that the probability of a user $i$ accessing the medium successfully is

$$
\operatorname{Pr}(\text { success })_{i}=\left(1-p_{p, i}\right) \times 0+\frac{p_{p, i} \times p_{i}}{\left(1-p_{i} \times p_{p, i}\right)} \times \prod_{j=1}^{n} p_{W, j} .
$$

Introducing $\hat{p}_{i}=p_{i} p_{p, i}$ and $\hat{g}=\prod_{j=1}^{n} p_{W, j}=\prod_{i=1}^{n}\left(1-\tilde{p}_{i}\right)$.

Note that, from (7) and $p_{p, i} \neq 0,1$, it follows that

$$
\begin{aligned}
& \hat{p}_{i} \neq 0,1 \quad \forall i \\
& \operatorname{Pr}\left(\text { success }_{i}=\frac{\hat{p}_{i}}{1-\hat{p}_{i}} \hat{g} .\right.
\end{aligned}
$$

Consequently, the fairness constraints can be represented as

$$
\begin{aligned}
& \frac{\operatorname{Pr}(\text { success })_{1}}{w_{1}}=\ldots \frac{\operatorname{Pr}(\text { success })_{i}}{w_{i}}=\ldots=\frac{\operatorname{Pr}(\text { success })_{n}}{w_{n}} \\
& \text { therefore } \frac{\hat{p}_{1} /\left(1-\hat{p}_{1}\right)}{w_{1}}=\ldots \frac{\hat{p}_{j} /\left(1-\hat{p}_{j}\right)}{w_{j}}=\ldots=\frac{\hat{p}_{n} /\left(1-\hat{p}_{n}\right)}{w_{n}} .
\end{aligned}
$$

The utility functions can also be rewritten as

$$
u_{i}=\frac{1}{p_{p, i}} \times \frac{\tilde{p}_{i}}{1-\tilde{p}_{i}} \times \hat{g} .
$$

Equations (30.a) and (30.b) are equivalent to (7) and (8), respectively. Equation (30.c) is equivalent to (11) due to the fact that $p_{p, i}$ is a positive constant. Therefore, from Theorem 7, we have that there is a unique set of solutions (in $\hat{p}_{i} \mathrm{~s}$ ) solving

$$
\frac{\partial u_{i}}{\partial \hat{p}_{i}}=0 \quad \forall i
$$

and

$$
\frac{\hat{p}_{1} /\left(1-\hat{p}_{1}\right)}{w_{1}}=\ldots \frac{\hat{p}_{j} /\left(1-\hat{p}_{j}\right)}{w_{j}}=\ldots=\frac{\hat{p}_{n} /\left(1-\hat{p}_{n}\right)}{w_{n}} .
$$

As $\hat{p}_{i}=p_{i} p_{p, i}$, it follows that there is unique set of $p_{i} \mathrm{~s}$ that will satisfy $\frac{\partial u_{i}}{\partial \hat{p}_{i}}=0$ and (30.b). 
Therefore, from Theorems 6 and 7, we have the following lemma:

\section{Lemma 11. CNE for (30.a)-(30.c) is unique.}

We now consider the throughput for this approximate case.

Lemma 12. Throughput is also optimized for the approximate case.

Proof. The throughput can be represented as

$$
\hat{\theta}=\sum_{i=1}^{n} p_{p . i} * p_{i} * \prod_{j \neq i}^{n} p_{W, j}=\sum_{i=1}^{n} p_{p . i} * u_{i} .
$$

As $p_{p, i}$ is constant, the proof follows exactly along the lines of Theorem 9 .

In this section, it was shown that the results for the complete information "Access Game" can be easily extended to the incomplete information "Access Game." Consequently, we can design a realistic medium access protocol as proposed in this paper. As shown, this protocol will guarantee fair share of bandwidth and optimal throughput.

\section{Conclusions}

We used Game Theory to address the issue of fair sharing of bandwidth in a distributed system with competitive users. The medium access was modeled as a noncooperative game, designated as the "Access Game." First, the "Access Game" for the complete information scenario was analyzed. It was proven that the CNE satisfying fairness is unique for the "Access Game" and this CNE optimizes the system throughput. Later, a simple mechanism was proposed to approximate an incomplete information scenario as a complete information scenario. The uniqueness and optimality of the CNE were proven for the approximate case also.

The proposed MAC scheme can be suitably applied for both wired and wireless local area networks. In busy systems like WLANs, the number of users changes frequently. Therefore, the optimal strategies for such systems would vary from one transmission slot to another. The proposed protocol computes optimal transmission probability for each slot. Therefore, it is an appropriate choice for WLANs. Moreover, for the approximation scheme presented in Section 4, we proposed that $R$ should broadcast the number of users present in the system at the beginning of a transmission slot. This technique can be incorporated with ease for the IEEE WLAN 802.11 MAC scenario. In wired LANs, the number of users does not change frequently and the assumption of complete information can be more easily applied. However, as our protocol computes optimal transmission strategies at the beginning of each transmission slot, there will be unnecessary computations for users. With small changes, the protocol can be applied in wired local area networks.

In all of the above discussions and analysis, it was assumed that the system is stable. However, the stability of the system would depend on the interaction between the packet arrival process and the performance of the MAC protocol [25]. Simply put, for stability, the number of unsuccessful packets should not become very large. The notion of stability has a direct bearing on the analysis and application of the protocol. As users choose a probabilistic transmission strategy in a given state, they would expect that state to repeat itself (assuming that the users play the "Access Game" a sufficient number of times). In other words, for the protocol to run correctly, the system should be ergodic. We are presently investigating this issue.

Most of the CSMA protocols in application today use window-based transmission strategies. The CSMA/CD version used by the Ethernet has a one persistent strategy for transmission and adopts a random exponential backoff strategy when collision occurs. The CSMA/CA strategy adopted for IEEE802.11 also uses a window-based strategy, but, philosophically, it is closer to the $p$-CSMA strategy as it does not transmit with probability " 1 " after sensing the medium idle. It would be useful to investigate whether the results of this paper can be extended to window-based schemes. Similar work has recently been published [37].

Finally, the issue of "cheating" users needs to be analyzed. If some users decide to deviate from the CNE operating point to some other selfishly beneficial operating point, what punitive strategies can other users take to bring the errant users back to the CNE operating point? We have formulated the problem in hybrid-control theoretic framework and are currently investigating suitable strategies to be adopted by users.

\section{ACKNOWLEDGMENTS}

This work was partially supported by the US National Science Foundation under Grant EIA 0086251 and the US Army Research Office under grant DAAD19-01-1-0502. The views and conclusions herein are those of the authors and do not represent the official policies of the funding agencies or the University of Central Florida. The authors are indebted to the reviewers for their constructive suggestions and helpful remarks.

\section{REFERENCES}

[1] J. Kruys, "HIPERLAN, Applications and Requirements," Proc. IEEE Int'l Symp. Personal Indoor and Mobile Radio Comm. (PIMRC '92), pp. 133-138, 1992.

[2] ETSI TS-RES 300 652, “Radio Equipment and Systems (RES); High Performance Radio Local Area Networks (HIPERLAN); Type 1; Functional Specifications," Oct. 1996.

[3] N. Abramson, "The ALOHA System-Another Alternative for Computer Communications," Am. Federation of Information Processing Socs. Conf. Proc., pp. 281-285, 1970.

[4] A.L. Garcia and I. Widjaja, Communication Networks. McGraw-Hill, 2000.

[5] G. Nutt and D. Bayer, "Performance of CSMA/CD Networks under Combined Voice and Data Loads," IEEE Trans. Comm., vol. 30, no. 1, pp. 6-11, 1982.

[6] G. Exley and L. Merakos, "Throughput-Delay Performance of Interconnected CSMA Local Area Networks," IEEE J. Selected Areas in Comm., vol. 5, no. 9, pp. 1380-1390, 1987.

[7] Y.-C. Liu and G. Wise, "Performance of a CSMA/CD Protocol for Local Area Networks," IEEE J. Selected Areas in Comm., vol. 5, no. 6, pp. 948-955, 1987.

[8] P. O'Reilly and J. Hammond Jr., "An Efficient Simulation Technique for Performance Studies of CSMA/CD Local Network," IEEE J. Selected Areas in Comm., vol. 2, no. 1, pp. 238-249, 1984. 
[9] T. Vo-Dai, "Throughput-Delay Analysis of the Nonslotted and Nonpersistent CSMA-CD Protocol," Local Computer Networks, P.C. Ravasio, G. Hopkins, and N. Naffah, eds., pp. 459-476, Amsterdam: North-Holland, 1982.

[10] M. Visser and M. El Zarki, "Voice and Data Transmission over an 802.11 Wireless Network," Proc. IEEE Int'l Symp. Personal Indoor and Mobile Radio Comm. (PIMRC), pp. 648-652, Sept. 1995.

[11] P802.11, IEEE Draft Standard for Wireless LAN Medium Access Control (MAC) and Physical Layer (PHY) Specification, D6.1, May 1997.

[12] IEEE 802.11e draft/D4.0, "Part 11: Wireless Medium Access Control (MAC) and Physical Layer (PHY) Specifications: Medium Access Control (MAC) Enhancements for Quality of Service (QoS)," Nov. 2002.

[13] A.B. MacKenzie and S.B. Wicker, "Stability of Multipacket Slotted Aloha with Selfish Users and Perfect Information," Proc. INFOCOM 2003, vol. 3, pp. 1583-1590, 2003.

[14] D. Fudenberg and J. Tirole, Game Theory. MIT Press, 1991.

[15] Y.A. Korilis, A.A. Lazar, and A. Orda, "The Designer's Perspective to Noncooperative Networks," Proc. INFOCOM '95, 1995.

[16] A. Orda, R. Rom, and N. Shimkin, "Competitive Routing in Multiuser Communication Networks," ACM/IEEE Trans. Networking, vol. 1, no. 5, pp. 510-521, Oct. 1993.

[17] E. Koutsoupias and C. Papadimitriou, "Worst-Case Equilibria," Proc. 16th Ann. Symp. Theoretical Aspects of Computer Science, pp. 404-413, 1999.

[18] T. Roughgarden and E. Tardos, "How Bad Is Selfish Routing?" J. ACM, vol. 49, no. 2, pp. 236-259, 2002.

[19] S. Shenker, "Making Greed Work in Networks: A Game-Theoretic Analysis of Switch Service Disciplines," Proc. SIGCOMM '94, 1994.

[20] A. Akella, S. Seshan, R. Karp, S. Shenker, and C. Papadimitriou, "Selfish Behavior and Stability of the Internet: A Game-Theoretic Analysis of TCP," Proc. SIGCOMM '02, Aug. 2002.

[21] C. Papadimitriou, "Algorithms, Games, and the Internet," Proc. ACM Symp. Theory of Computing (STOC), 2001.

[22] L. Kleinrock and F. Tobagi, "Packet Switching in Radio Channels: Part I-Carrier Sense Multiple-Access Modes and Their Throughput-Delay Characteristics," IEEE Trans. Comm., vol. 23, no. 12, pp. 1400-1416, 1975.

[23] D. Bertsekas, Nonlinear Programming. Athena Scientific, Sept. 1999.

[24] D. Qiao and K.G. Shin, "Achieving Efficient Channel Utilization and Weighted Fairness for Data Communications in IEEE 802.11 WLAN under the DCF," Proc. 10th Int'l Workshop Quality of Service (IWQoS 2002), May 2002.

[25] G. Fayolle, E. Gelenbe, and J. Labetoulle, "Stability and Optimal Control of the Packet Switching Broadcast Channel," J. ACM, vol. 24, pp. 375-386, July 1977.

[26] J. Nash, "Non-Cooperative Games," Annals of Math., vol. 54, pp. 286-295, 1951.

[27] J. Harsanyi, "Games with Incomplete Information Played by Bayesian Players," Management Science, vol. 14, pp. 159-182, 320334, 486-502, 1967-1968.

[28] J. Rosen, "Existence and Uniqueness of Equilibrium Points for Concave n-Person Games," Econometrica, vol. 33, pp. 520-534, July 1965.

[29] A. Demers, S. Keshav, and S. Shenker, "Analysis and Simulation of a Fair Queueing Algorithm," ACM SIGCOMM Computer Comm. Rev., vol. 10 no. 4, pp. 1-12, Sept. 1989.

[30] S. Rakshit and R. Guha, "Optimal Mac Protocols," Proc. Int'l Computing Conf., 2004.

[31] V. Bharghavan, A. Demers, S. Shenker, and L. Zhang, "MACAW: A Media Access Protocol for Wireless LANs," Proc. ACM SIGCOMM, pp. 212-225, Aug. 1994.

[32] M. Gerla, K. Tang, and R. Bagrodia, "TCP Performance in Wireless Multi-Hop Networks," Proc. IEEE Workshop Mobile Computing Systems and Applications (WMCSA), pp. 41-50, Feb. 1999.

[33] G.L. Choudhury and S.S. Rappaport, "Priority Access Schemes Using CSMA-CD," IEEE Trans. Comm., vol. 33, pp. 620-626, July 1985.

[34] S.M. Sharrock and D.H. Du, "Efficient CSMA/CD-Based Protocols for Multiple Priority Classes," IEEE Trans. Computers, vol. 38, pp. 943-954, July 1989.

[35] N.H. Vaidya, P. Bahl, and S. Gupta, "Distributed Fair Scheduling in a Wireless LAN," Proc. MOBICOM, 2000.

[36] S. Rakshit and R. Guha, "A Non-Cooperative Game-Theoretic Model for the Medium Access and Fair Bandwidth Sharing in WLAN," Wireless Comm. and Mobile Computing, in revision.
[37] M. Cagalj, S. Ganeriwal, I. Aad, and J.P. Hubaux, "On Cheating in CSMA/CA Ad Hoc Networks," Proc. Infocom, 2005.

[38] L. Kleinrock, Queueing Systems Vol. 2: Computer Applications. New York: John Wiley \& Sons, 1976.

[39] A. Parekh and R. Gallager, "A Generalized Processor Sharing Approach to Flow Control in Integrated Services Networks: The Single Node Case," IEEE/ACM Trans. Networking, vol. 1, no. 3, pp. 344-357, June 1993.

[40] J.C.R. Bennet and H. Zhang, "WF2Q: Worst-Case Fair Weighted Fair Queueing," Proc. IEEE INFOCOM '96, Mar. 1996.

[41] F.A. Tobagi, "Carrier Sense Multiple Access with Message-Based Priority Functions," IEEE Trans. Comm., vol. 30, no. 1, pp. 185-200, Jan. 1982.

[42] E. Altman and R. El Azouzi, "Constrained Traffic Equilibrium in Routing Networks," IEEE Trans. Automatic Control, vol. 48, no. 9, pp. 1656-1660, Sept. 2003.

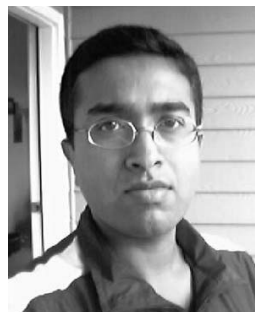

Sudipta Rakshit is a $\mathrm{PhD}$ candidate in the School of Computer Science in the College of Engineering and Computer Science at the University of Central Florida. He is expected to graduate in August 2005.

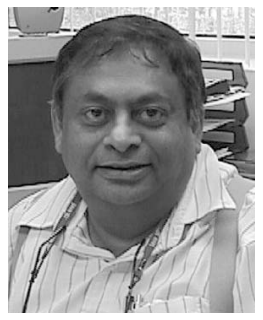

Ratan K. Guha received the BSc degree with honors in mathematics and the MSc degree in applied mathematics from Calcutta University and received the $\mathrm{PhD}$ degree in computer science from the University of Texas at Austin in 1970 . He is currently a professor of computer science at the University of Central Florida, Orlando. His research interests include distributed systems, computer networks, security protocols, modeling and simulation, and computer graphics. He has authored more than 100 papers published in various computer journals, book chapters, and conference proceedings. His research has been supported by grants from the US Army Research Office, US National Science Foundation, STRICOM, PM-TRADE, and the State of Florida. He has served as a member of the program committee for several conferences, as the general chair of CSMA '98 and CSMA 2000, and as the guest coeditor of a special issue of the Journal of Simulation Practice and Theory. He is a member of the ACM, IEEE, IEEE Computer Society, and SCS and is currently serving as a member of the Board of Directors of SCS.

$\triangleright$ For more information on this or any other computing topic, please visit our Digital Library at www.computer.org/publications/dlib. 